\title{
Anatomic substrate to pain originating in the disc and chronic low back pain posture damage resolution
}

\author{
Alfredo Romeo, ${ }^{1}$ Marianna Chierchia, ${ }^{2}$ Carolina Baiano, ${ }^{1}$ Dante Ronca ${ }^{2}$ \\ 'Department of Physical Medicine and Rehabilitation, Umberto I Hospital, Siracusa; \\ 2 Multidisciplinary Department of Medical, Surgical and Dental Specialties, Second Orthopedic \\ Clinic, Second University of Naples, Naples, Italy
}

\begin{abstract}
Chronic low back pain is a disease that most often affects people between 45 and 64 years. The incidence of low back pain goes from 60 to $90 \%$ and the annual incidence is $5 \%$. Often low back pain is accompanied or replaced by an irradiated lower limb pain, configuring the symptomatology of lumboradiculalgia, whose most frequent cause is the disco-radicular conflict. The cause of the onset of pain, even excruciating, in cases of radicular compression of disc origin is not yet clear. Low back pain of disc origin is thought to be caused by direct mechanical compression factors; by indirect mechanical factors ischemia or venous stasis of vasa nervorum; and by type of immune-mediated inflammatory factors (reaction hernia) and/or biochemical evidence related to the disc type (the presence of substances which induce the inflammatory reaction). When a patient is suffering by chronic low back pain, apart from the algic problem, a series of changes in posture will occur, which contribute to worsen low back pain itself. The effects of a polluted posture and, therefore, the muscle retraction manifest in the joints in the form of compression, axial rotation and translation, resulting in modifications of the skeletal morphology (scoliotic attitudes, hyperkyphosis, valgus and varus of the knee, etc.) and possibly evolving into important postural
\end{abstract}

Correspondence: Marianna Chierchia, Multidisciplinary Department of Medical, Surgical and Dental Specialties, Second Orthopedic Clinic, Second University of Naples, via Luigi De Crecchio 6, 80130 Naples, Italy.

E-mail: mariannachierchia@virgilio.it

Conflict of interest: the authors declare no potential conflict of interest.

Key words: Chronic low back pain; Secondary posture damage; Percutaneous paravertebral intramuscular $\mathrm{O}_{2}-\mathrm{O}_{3}$.

Received for publication: 16 November 2016.

Accepted for publication: 13 December 2016.

(C) Copyright A. Romeo et al., 2016

Licensee PAGEPress, Italy

Ozone Therapy 2016; 1:6472

doi:10.4081/ozone.2016.6472

This article is distributed under the terms of the Creative Commons Attribution Noncommercial License (by-nc 4.0) which permits any noncommercial use, distribution, and reproduction in any medium, provided the original author(s) and source are credited. disorders. Currently, the treatment of chronic low back pain and postural secondary disorders is still an open problem. The aim of this preliminary study is: i) to highlight the high incidence of low back pain by providing evidence that there is an anatomical substrate to low back pain originating in the disc; ii) and to assess the effects of the use of oxygen-ozone percutaneous paravertebral intramuscular therapy in synergy with a functional rehabilitation program in a group of patients suffering from chronic low back pain. From the data obtained, the combination of percutaneous paravertebral intramuscular oxygen-ozone therapy with functional rehabilitation is confirmed as an effective and safe method, whose favorable results are evident at the level of lumbar pain symptoms, postural secondary abnormalities, disability in activities of daily living, the mood and tone of the psychological state.

\section{Introduction}

Vertebral pain has a great incidence and its social and economical impact is extremely high. It represents the most common musculoskeletal disease among the adult population as it represents more than the $50 \%$ of all musculoskeletal diseases.

Several studies suggest that the $80 \%$ of the population will suffer from variable degree low back pain within one year, and the $30 \%$ of them will be treated for this kind of illness.

Sometimes vertebral low back pain syndrome, which can leads to irritative symptoms of nerve roots, is used to recur after an acute episode, becoming a chronic or subacute syndrome with serious disabilities on the job and everyday life.

Indeed, chronic low back pain represents the most common cause of limitation of population activities ranging from 45 to 65 years old.

Spine pain originating in the disc is thought to be due to different compression direct mechanical factors; indirect ischemia mechanical factors or Vasa nevrorum venous stasis; immune-mediated inflammatory factors (reaction to hernia); and/or biohumoral inflammatory factors associated with the disc (substancies that induce the inflammatory reaction).

When a patient suffers from chronic low back pain a serious of posture modifications occur and they worsen lower back pain. Generally, besides the biomechanical factors, a huge amount of psychological and social problems contributes to worsen the pain.

The aim of this preliminary study is to highlight: i) high low back pain incidence proving the existence of a subatomic substrate of the pain originating in the disc; ii) valuing the effect of the percutaneous, paravertebral, intramuscular oxygen-ozone treatment combined with a functional rehabilitation program in a group of patients affected by chronic low back pain. 


\section{Materials and Methods}

In this regard a two arm observational study was conducted in an outpatient setting. A cohort of 84 people suffering from chronic low back pain and symptoms related to deep posture alterations was chosen.

We randomly subdivided subjects into two groups by age and clinical features (Table 1): the first, group A, made of 41 patients, was treated with $\mathrm{O}_{2}-\mathrm{O}_{3}$ treatment and they underwent a specific rehabilitation program for a period of 8 consecutive weeks (4 sessions/week), while the second, group $\mathrm{B}$, made of 43 patients underwent only $\mathrm{O}_{2}-\mathrm{O}_{3}$ treatment.

The therapeutic table expected: 12 sessions of low back intramuscular paravertebral oxygen-ozone treatment, with a two week iteration for the first 8 sessions, one week iterations for the other 4 sessions. We used $22 \mathrm{G}$ needles (for well-built subjects), 23G (for feminine and thin subjects); we injected 10 cc of oxygen-ozone on each side, at the concentration of 20-25 g-mL, following the rules given by the Scientific Society: infiltrate $2 \mathrm{~cm}$. From the spinous apophisys near the interested space, double aspiration, light massage, supine position for at least 10 minutes after the infiltration. ${ }^{1}$

The rehabilitation process is organized according to an initial questionnaire (outcome measurement), muscular resistance measurement, range of motion (ROM), posture tests, medical history of the patient.

The rehabilitation program consisted in stretching exercises, range of motion, and muscle strengthening, in addition we have been proposed proprioceptive and coordination exercises and muscle resistance. The treatment includes controlled movements in lateral lumbar/dorsal and/or cervical flexion, and movements in flexion, extension and rotation. Every session is preceded by aerobic warming up of about $10 \mathrm{~min}$. A series of myofascial relaxing and stretching exercises have been proposed after every therapeutic exercise session. ${ }^{2-4}$

The rehabilitation begins with low loads for the first weeks, in order to improve the range of motion and to teach a correct coordination and spine control. Loads are gradually intensified according to the gradualness principle until when, by the end of program, patients are instructed on how to continue a secondary individual prevention program (once or twice per week).

This posture-education program ended up with the total mobility restoration and the correct spine posture, in addition to the torso muscle balance. A key factor to chronic lumbar pain management is to instruct the patient and making him aware of his pathology, in order to promote self-management of the therapy and the correction of wrong habits.

Patients chosen for the treatment underwent the enrollment act (t0), 4 weeks (t1), 8 weeks (t2), 6 months ( $\mathrm{t} 3$ ), physiatric assessment and administration of the following ratios as outcome measurements: visual analogue scale (VAS), SF-36, Oswestry Low Back Pain Disability Questionnaire and Roland Morris Disability Questionnaire. Roland Morris Disability Questionnaire is a specific disability scale for each pathology that assesses functional status for patients suffering from spine or lower limb pain: score range from 0 to 23. Meanwhile, pain intensity is assessed with a VAS in which 0 represents no pain and 10 represents the most intense pain ever.

Table 1. Characteristics of the patients who underwent the $\mathrm{O}_{2}-\mathrm{O}_{3}$ treatment.

\begin{tabular}{lcc}
\hline Patients' characteristics & Group $\mathrm{A}(\mathrm{N}=41)$ & Group $\mathrm{B}(\mathrm{N}=\mathbf{4 3})$ \\
Age (years) & $49.82 \pm 10.23$ & $45.88 \pm 9.24$ \\
Sex & & \\
Females (n) & 25 & 28 \\
Males (n) & 16 & 15 \\
\hline Duration of symptoms(weeks) & 28 & 25 \\
\hline
\end{tabular}

SF-36 is a generic questionnaire about health status that consists of 36 items about physical and social conditions subdivided into 8 quality domains. Score, that range from 0 to 100 , indicates less serious symptoms for progressively growing values.

Oswestry Low Back Pain Disability Questionnaire and Roland Morris Disability Questionnaire are extremely important documents that assess functional disability and limitations in everyday life in patients affected by low back pain.

One of the major problems for affected patients is the considerable reduction in the torso and pelvis ROM, in addition to flexion and extension movements limitation in the coxofemural articulation. For this reason have been considered: i) lumbosacral spine ROM assessment; ii) patient satisfaction degree about the efficacy of the treatment was expressed through judgment.

A set of quick use tests, with a certain scientific trust, have been used for posture assessments (Fukuda Test, Forward Bending Test, Breech Convergence Maneuver).

The analysis of the results (before and after the treatment), was conducted intragroup by Wilcoxon Test and intergroup with Mann-Whitney Test (Statistical significance $\mathrm{P}<0.05$ ).

0xygen ozone is generated by MEDICAL 99IR with photometric system manufactured by Multiossigen.

\section{Results}

Both groups patients have observed statistically significant results, group $\mathrm{A}$, in particular, treated with $\mathrm{O}_{2}-\mathrm{O}_{3}$ in addition to therapeutic exercise reported a greater significance for all scales of values with a $\mathrm{P}<0.005$ significance, in $75 \%$ of patients. However, the group who underwent only $\mathrm{O}_{2}-\mathrm{O}_{3}$ treatment showed important results too, with $\mathrm{P}<0.05$ significance although it still remains statistically below group A according to the total score of valuation scales SF-36, Oswestry, Roland and Morris (Figures 1-3).

The parameters obtained from the statistical analysis showed a greater reduction in pain symptoms, disability and quality of life and a better resolution of secondary posture damage in subjects who received a specific rehabilitative treatment rather than the group who underwent only $\mathrm{O}_{2}-\mathrm{O}_{3}$ treatment.

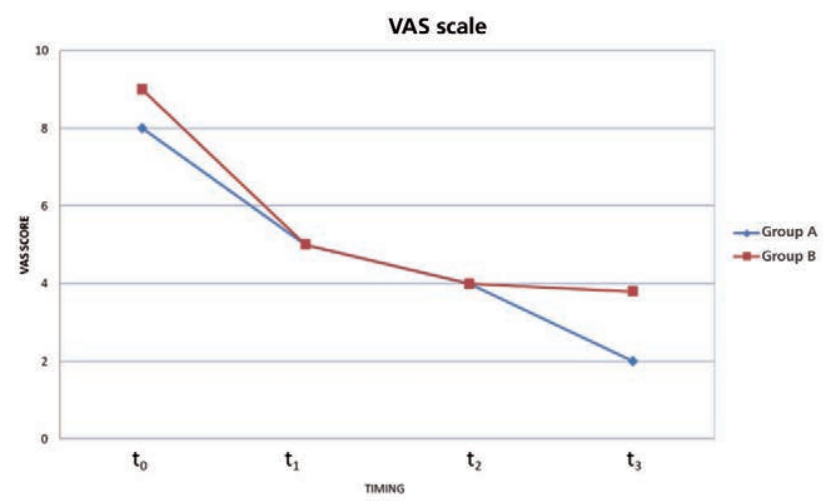

Figure 1. Group A showed a very early reduction in pain: as early as $T 1$ pain has dropped in the $40 \%$ of patients $(P<0.05)$; the decrease continued regularly in all the subsequent controls $(\mathrm{P}<0.005)$. Compared to base time at 6 months $(\mathrm{T} 3>\mathrm{T} 0)$ the pain score fell in the $75 \%$ of the patients. Also control group B showed a clear improvement. When compared, group $A$ had better results than group $\mathrm{B}$ in all follow-ups with $\mathrm{P}<0.05$. 


\section{Discussion}

Chronic low back pain is the most common cause of long term disability that affects mid adult subjects (from 45 to 64 ). The incidence of low back pain ranges from 60 to $90 \%$, while the annual incidence is about $5 \%$. Its prevalence varies depending on the age. Indeed, it is low (4-18\%) between 20 and 24 years old, it is high (8-32\%) between 55 and 64. ${ }^{5,6}$

In USA 5.2 millions of inhabitants have a disability that is secondary to chronic low back pain. It is permanent for half of them.

The economical impact coming from this kind of pathology is extremely important, indeed, spine diseases occupy the fifth place, among all the pathologies, regarding health care costs and the first regarding absenteeism in the workplace.

Among spine pains, low back pain is a common symptom and it can cause pain that radiates to lower limbs (lumbar radiculopathy-sciatica, lumbar cruralgia). ${ }^{7}$

It is a very complex disease because it can be caused by different anatomic structures: i) posterior longitudinal ligament; ii) anterior longitudinal ligament; iii) interspinous ligaments; iv) supraspinous ligaments; v) yellow ligament; vi) joint capsule; vii) epyphiseale plate; viii) annulus fibrosus; ix) nucleus polposus.

The majority of those tissues that surround the vertebral disc have nociceptive nerve endings, thus, they are source of pain. Intervertebral discs are innerved only at the periphery. Free nociceptive endings are located in the surface layer (laminae) of the annulus fibrosus until 1-2 $\mathrm{cm}$ depth. The two sinu-vertebral nerves provide two branches to 2 discs near. Other fibers, possibly amyelinic, participate in the innervations of the anterior portion of the disc.

The nucleus polposus is the only structure not having a sensory innervation.

Spine innervation is metameric and it is due to 2 segmental nerves, Luschka sinu-vertebral nerve and the dorsal root of the spinal nerve. Luschka sinu-vertebral nerve is distributed to the annulus, intraspinal fibrousus formations, sinus venous and the dura mater. The dorsal root is distributed to the outer periosterum, zigoapofisary joints and to the posterior ligaments of the spine; it is much smaller than the anterior root that participate in the formation of the lumbar plexus. ${ }^{8,9}$

With regard to the vertebral canal and its content, the majority of disagreements about the presence and the nature of sensory receptors are due to different staining methods, indeed, gold chloride staining method appears more specific than methylene blue one. We can find 3 kinds of nerve endings: free nerve endings, which are triggered by painful stimuli; Pacinian corpuscles and Ruffini's corpuscles, which are encapsulated mechanoreceptors, and they respond to proprioceptic sensory.

All juxta-discs structures can induce to discal pain, but annulus fibro- sus and the Longitudinal ligament are the main responsible for this kind of pain because zigoapofisary joints stimulation would cause low pain.

Low back pain is often substituted or goes with a pain radiating to the lower limb, configuring picture symptoms of lomboradicolopatia compression of spine cord nerves. Its most common cause is a discradicular conflict. The cause of the onset of pain, which can also be stabbing, in cases of radicular compression originating from the disc is not well clear. ${ }^{10-12}$

Indeed, compression of a root causes a double effect, mechanical direct of the nerve fibers and indirect through the reduction or the interruption of the emetic circle on the nerve tissue. It has been proved that nerve flow may also be kept under strong compression as long as a regular concentration of oxygen is kept. On the contrary, in case of hypoxia it is noticeable a fast deterioration, even without compression. All of this suggests that an ischemia results in much more serious nerve flow deterioration than the compression itself. ${ }^{13-14}$

Another source of pain is inflammation. Indeed, a phospholipid activity $\mathrm{A}_{2}$ has been noticed in human disc fragments coming from discectomy, it is a main element in the production of inflammatory mediators (leukotriene and prostaglandin). In the patho-physiology of radic-

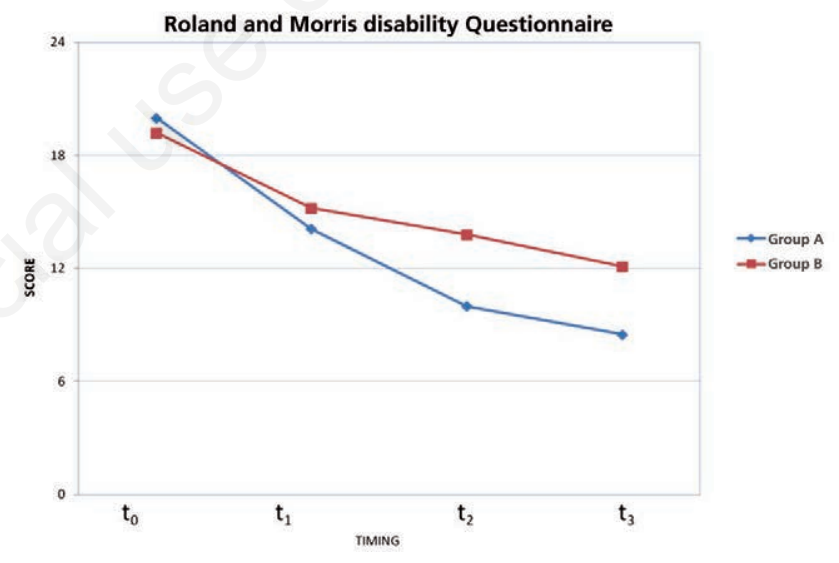

Figure 2. Group A: values improved rapidly after 4 (T1) and 8 (T2) weeks with significant decrease at T3 with $\mathrm{P}<0.05$ in both follow-ups. Group B: variations at the various follow-ups were of minor entity, therefore, the difference was significant only at 6 months (T3). When compared, group A still had better results than group $B$ at $\mathrm{T} 2$ and $\mathrm{T} 3$ follow-ups with $\mathrm{P}<0.05$.

\section{Oswes try Low Back Pain Dis ability Questionnaire}

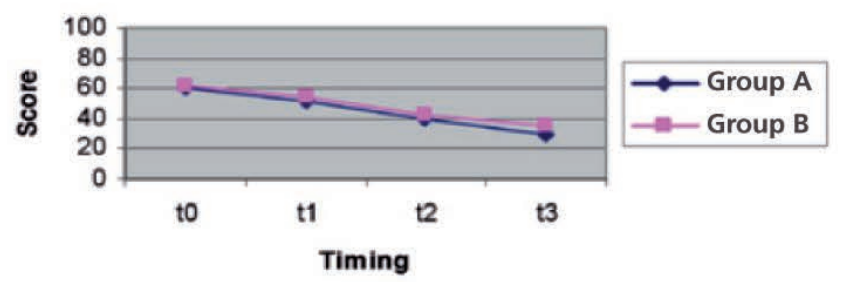

Short-Form Health Survey (SF-36)

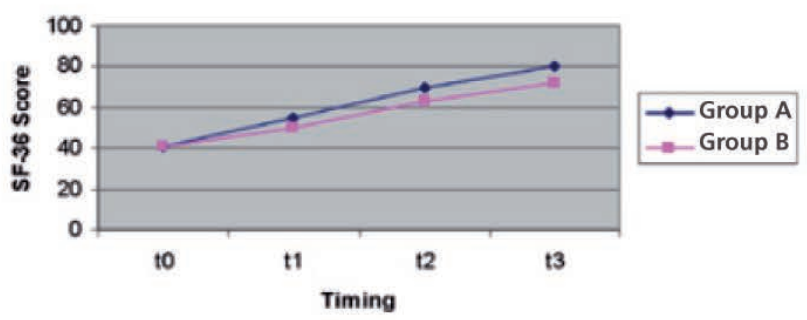

Figure 3. According to the total score of evaluation scales Short Form Health Survey-36 and Oswestry Low Back Pain Disability Questionnaire, disability and quality of life have a better resolution (with $\mathrm{P}<0.05$ ) in subjects who received also a specific rehabilitative treatment (group A) rather than the group who underwent only $\mathrm{O}_{2}-\mathrm{O}_{3}$ treatment (group $\mathrm{B}$ ). 
ular suffering it's important to understand not only a mechanism of axonal compression but also an alteration of microcirculation besides inflammatory and scar reactions. ${ }^{15}$

All this confirms the existence of an anatomic-biochimical substrate to pain of discal origin. ${ }^{16}$

When a patient suffers from chronic low back pain a serious of posture modifications is evident. Spine short muscles (short rotators, interspinous and interapophyseal muscles) play an important role in the origin of low back pain and the posture alteration deriving from it. Indeed, those muscles contract themselves because of a painful reflection effect conveyed by the external branches of posterior ramus of spinal nerves, thus determining muscular tissue ischemia that leads to pain. Reflex pain only intensify muscle contracture ending up with a vicious circle. ${ }^{17}$

Also muscles such as Piriformis muscles, Psoas muscle, Quatratus Lomborum and Paravertebral muscles are potentially implicate in the origin of low back pain both acute and chronic. Psoas muscle in particular is a mainstay muscle with some unique features. It crosses axial skeleton up to the lower limb, where it meets the little trochanter. It exercises its influence the hip and the lumbar column the most, where it acts as a posture muscle. From an anatomic and a biomechanical point of view, it works in association with the spinal unit (vertebra and disc) and it is thought to increase the intradiscal pressure.

Some authors show a decrease in the psoas sectional area bilaterally in presence of a lateralized herniated disc. Such reduction is positively correlated with the duration of sciatica.

The effects of a wrong posture and muscular retraction occur at the joints in form of compression, axial rotation and translation, thus resulting in changes in skeletal morphology (scoliosis attitude, Hyperkyphosis, knee Valgus and Varus deformity, etc.) and they can also evolve into posture disorders up to real diseases. The pain causes: antalgic contraction of paravertebral muscles with smoothing of lumbar lordosis; furthermore if the muscular retraction is asymmetric this leads to the development of a scoliosis attitude, prepending spine with the consequent pelvis nutation.

The majority of patients suffering from chronic low back pain shows a deconditioning syndrome: ${ }^{18,19}$ a reduction in physical activity can lead to a reduce joint function, a loss of strength, resistance and muscular coordination. It was proved that low back pain affected patients' paravertebral muscles are less developed, they contain more fat and show a selective muscular fibers atrophy. ${ }^{20-23}$ Several studies on chronic low back pain have highlighted paravertebral muscles reduced in peak force and resistance. ${ }^{23-25}$ Deconditioning contributes in worsening the pain and negatively affects postures that the patient adopts in order to avoid the pain.

Therefore, patients enter a vicious circle worsening their physical condition. This model suggests a correlation between the physical condition undermining and the disease behavior in the chronic pain.

Therefore, in patients with low back pain, injury does not represent the main problem anymore, but it is also important a rehabilitation able to assist completely the patients, avoiding a permanent disability.

Nowadays the treatment of chronic low back pain is still a problem, because it is resistant to the common pharmaceutical therapy and also because some of the proposed treatments do not exist so far. All the proposed therapies have to compare themselves to a multiform and multifactorial disease, in which inflammatory, biomechanical, postural and psycho-social factors are combined. Indeed, patients suffering from this disease show a big amount of psychological and social problems other than biomechanical/postural factor. All that contributes in keeping the pain. That is the reason why a multidisciplinary integrated approach, involving various aspects of the subject, is useful for this kind of patients. ${ }^{3,4}$

In our experience the association oxygen-ozone paravertebral therapy combined with a functional rehabilitation happened to be a very effective and safe method. Its results resulted undeniable in terms of lower back pain symptoms, secondary posture modifications, disability in activities of daily living (ADL), mood and psychological status. , $16,26-30^{-1}$

Ozone therapy (the oxygen-ozone mixture) represents more and more an efficient solution for the chronic low back pain conservative treatment, that is because it is proved not to have side-effects and it shows an analgesic and anti-inflammatory efficient effect.

Those effects are due to the pro-inflammatory prostaglandin inhibition of the synthesis; to the inhibition of the bradykinin release or pain compounds; to the neutralization of oxygen reactive species in order to stimulate the local production of antioxidant enzymes; to a modulation in the production of pro-inflammatory cytokine.

The gas mixture reduces the pain helping to develop the local microvascularization and remove the inflammatory substances thanks to the link between ozone and unsaturated fatty acids and erythrocytes peroxides. It increases the permeability of the erythrocytes membrane improving the perfusion of $\mathrm{O}_{2}$ to the textures and the metabolic exchanges.

Moreover, the improved local vascularization obtained thanks to the oxygen-ozone, together with the spine mobilization due to the functional rehabilitation, seems to be able to stop the pathophysiological mechanism of the discal degeneration.

It is important to remember, in addition to what noticed so far, the $\mathrm{O}_{2}-\mathrm{O}_{3}$ mixture tension-relief effect along with reflexology action on trigger points.

Patients affected from low back pain treatment aims to: i) reducing/eliminating the pain; ii) restoring a normal joint and posture function; iii) reducing the need of medical therapies over time; iv) allowing a regular lifestyle..$^{29,30}$

\section{Conclusions}

The results of our study show that percutaneous paravertebral intramuscular $\mathrm{O}_{2}-\mathrm{O}_{3}$ treatment along with a functional rehabilitation program improves the symptoms and deficit in low back pain affected patients. The authors aim to continue their study by monitoring long term modification of the obtained results, in order to better understand the way to modulate the disability in such a common and chronic-progressive disease.

\section{References}

1. Romeo A, Cirillo F. [Contributo della chinesiterapia e dell'ossigenoozono terapia nel trattamento dei "conflitti" disco-radicolari lombosacrali]. [Article in Italian]. Rivista di Neuroradiologia 2001;14:47-9.

2. Manniche C, Hesselsoe G, Bentzen L, et al. Clinical trial of intensive muscle training for chronic low-back pain. Lancet 1988;2:1473-6.

3. Negrini S, Paroli C. [La riabilitazione nelle sindromi algiche lombari. La riabilitazione in Reumatologia]. [Book in Italian]. Dott. G. Arioli. Fidenza: Ed. Mattioli; 2001.

4. Altmaier EM, Lehmann TR, Russell DW, et al. The effectiveness of psychological interventions for the rehabilitation of low back pain: a randomized controlled trial evaluation. Pain 1992;49:329-35.

5. Cats-Baril WL, Frymoyer JW. Identifying patient at risk of becoming disabled because of low-back pain. Spine 1991;16:605-7.

6. Delauche-Cavallier MC, Budet C, Lardo JD, et al. Lumbar disc herniation. Computed tomography scan changes after conservative treatment of nerve root compression. Spine 1992;17:927-33.

7. Frymoyer JW. Back pain and sciatica. New Engl J Med 1988;318: 291-300.

8. Groen GJ, Baljet B, Drukker J. Nerves and nerve plexuses of the human vertebral column. Am J Anat 1990;188:282-96. 
9. Heliivaara M, Mäkelä M, Knekt P, et al. Determinants of sciatica and low-back pain. Spine 1991;16:558-68.

10. Wong DA. Mcnab's backache. Baltimore: Lippincott Williams \& Wilkins; 1977.

11. Postacchini F, Lami R, Pugliese 0. Familial predisposition to discogenic low-back pain. An epidemiologic and immunogenetic study. Spine 1988;13:1403-5.

12. Putti V. New concepts in the pathogenesis of sciatic pain. Lancet 1927;2:53-60.

13. Saal JA, Saal JS, Herzog RJ. The natural history of lumbar intervertebral disc extrusions treated nonoperatively. Spine 1990;7:683-96.

14. Spengler DM, Bigos SJ, Martin NA, et al. Back injuries in industry: a retrospective study. Spine 1986;11:241-5.

15. Virgin WJ. Experimental investigations into the physical properties of the intervertebral disc. J Bone Joint Surg Brit 1951;33:607-11.

16. Simonetti L, Agati R. Why does disc-root conflict generate pain. Neuroradiology1998;11:403-4.

17. Pacini T. [Studio della postura e indagine baropodometrica]. [Book in Italian]. Florence: Chimat; 2000.

18. Kienerman L, Slade PD, Stanley IM, et al. The prediction of chronicity in patients with an acute attack of low back pain in a general practice setting. Spine 1995;30:478-84.

19. Mayer TG, Smith SS, Keeley J, Mooney V. Quantification of lumbar function. Part 2: sagittal plane trunk strength in chronic low-back pain patients. Spine 1985;10:765-72.

20. Cooper RG, St Clair Forbes W, Jayson MI. Radiographic demonstration of paraspinal muscle wasting in patients with chronic low back pain. Brit J Rheumatol 1992;31:389-94.

21. Fidler MV, Jowett RL, Troup JD. Myosin ATPase activity in multifidus muscle from cases of lumbar spinal derangement. J Bone Joint Surg 1975;57:220-7.
22. Hadar H, Gadoth N, Heifetz M. Fatty replacement of lower paraspinal muscles: normal and neuromuscular dìsorders. Am J Rheumatol 1983;141:895-8.

23. Lultman G, Nordin M, Saraste H, Ohlsen H. Body composition, endurance, strength, cross- sectional area and density of muscle erector spinae in men with and without low back pain. J Spinal Disord 1993;6:114-23.

24. Addison R, Schultz A. Trunk strengths in patients seeking hospitalization for chronic low-back disorders. Spine 1980;5:539-44.

25. Bierìng-Sòrensen E. Physical measurements as risk indicator for low back trouble overone-year period. Spine 1984;9:106-19.

26. Peul WC, van Houwelingen HC, van denHout WB, et al. Surgery versus prolonged conservative treatment for sciatica. New Eng J Med 2007;356:2245-56.

27. Arena M, Savoca G, Papa R. [Infiltrazione percutanea paravertebrale di 0203 e trattamenti integrati nella terapia delle ernie e dei conflitti disco articolari a sede lombo-sacrale]. [Article in Italian]. Rivista Italiana di Ossigeno-Ozonoterapia 2005;4:131-2.

28. Coclite D, Napoletano A, Barbina D, et al. [Ossigeno-ozono terapia nel trattamento delle lombosciatalgie da ernia discale con tecnica iniettiva intramuscolare paravertebrale]. [Contribution in Italian]. Proceedings of the Consensus Conference. Roma: Istituto Superiore di Sanità; 2006.

29. Iliakis E. [Utilizzo dell'ossigeno-ozono terapia nella pratica ortopedica]. [Article in Italian]. Acta Toxicol Ther 1996;17:2-3.

30. Paoloni M, Di Sante L, Cacchio A, et al. Intramuscular oxygenozone therapy in the treatment of acute back pain with lumbar disc herniation: a multicenter, randomized, double-blind, clinical trial of active and simulated lumbar paravertebral injection. Spine $2009 ; 34: 1337-44$. 